\title{
FACE RECOGNITION IMPLEMENTATION ON RASPBERRYPI USING OPENCV AND PYTHON
}

\author{
Manav Bansal \\ Sir Chhotu Ram Institute of Engg. and Tech., \\ CCS University, Meerut, Uttar Pradesh, India
}

\begin{abstract}
Distinguishing an individual with a picture has been advanced through the broad communications. Be that as it may, it is less powerful to unique finger impression or retina examining. This report depicts the face detection and recognition smaller than normal task attempted for the visual observation and self-governance module at Plymouth college. It reports the innovations accessible in the Open-Computer-Vision (OpenCV) library and technique to execute them utilizing Python. For face identification, Haar-Cascades were utilized and for face recognition Eigenfaces, Fisherfaces and Local double example histograms were utilized. The procedure is portrayed including stream diagrams for each phase of the framework. Next, the outcomes are indicated including plots and screen-shots pursued by an exchange of experienced difficulties. The report is finished up with the creators' feeling on the venture and potential applications. This paper means to execute a face recognition programming code dependent on the strategy for Haar Cascade Classifiers and to effectively actualize this code on the Raspberry Pi stage for continuous recognition. In this paper, an endeavor to execute face acknowledgment calculation on an equipment stage, which is basic, yet productive in utilization is taken up. The product source codes for both detection and recognition of countenances are composed utilizing Opencv and Python.
\end{abstract}

Key words: Face Recognition, Open-CV, Raspberry-pi.

Cite this Article: Manav Bansal, Face Recognition Implementation on RaspberryPi using OpenCV and Python, International Journal of Computer Engineering and Technology 10(3), 2019, pp. 141-144.

http://iaeme.com/Home/issue/IJCET?Volume=10\&Issue=3

\section{INTRODUCTION}

Face detection nowadays is generally satisfactory because of high exactness and uniqueness over biometric framework like unique finger impression or iris acknowledgment. The human face assumes a significant job in our everyday life, passing on individuals distinguish. Each individual has their very own face character. This paper utilizes strategy which absolutely autonomous on outward appearance which influences on acknowledgment framework. Web cam snap a photo and utilizing face descriptor apparatus face acknowledgment finished with database which previously put away on raspberry pi [1] and data showed on yield screen. 
Face descriptor apparatus utilized for face acknowledgment with three perspectives as recognize a face to follow, distinguish facial element to track and track the face [4] Feature extraction is dimensionality decrease system. This methodology is helpful when picture size are bigger. Fundamental point of highlight extraction is to speak to the data of unique picture in lower dimensionality space with tasks, for example, edge detection, corner detection of picture. Normal programming language programming, for example, matlab give include extraction system. Highlight extraction systems utilizes less power, less memory and furnish yield with adequate exactness [3].web cam's pictures as R,G,B (red, green, blue ) appeared in fig. 1 This hued picture containing R, G, B segments and for edge coordinating strategy convert this shaded picture to dim picture. 8 bit hued picture has 256 shades of every R, G, B, so it builds the multifaceted nature of methods. Convert RGB qualities to ycbcr colour space $(y=$ luminance and chrominance $=c b$ and $c r)$. To get genuine picture size ascertain the most extreme and least of the picture. During this methodology unique picture can be obscured to anticipate this issue lightning pay strategy used.Blob detection technique is utilized for recognizing diverse locale of face that contrast in properties, for example, splendor or shading when contrasted with encompassing area. As indicated by face descriptor device convert hued picture to dim picture in which skin part made white and non-skin part, for example, eye and mouth is obscured appeared in fig. 2 and by utilizing measurement of dim picture identify and perceive the picture. Dim pictures utilizes in the middle of 0 and 1 pixels consequently computation is simple and unpredictability is diminished when contrasted with hued picture.

\section{IMPLEMENTATION}

The Raspberry Pi is a progression of little single-board PCs created in the United Kingdom by the Raspberry Pi Foundation to advance the educating of fundamental software engineering in schools and in creating nations. The first model wound up unquestionably more famous than foreseen, selling outside of its objective market for utilizations, for example, mechanical autonomy. Peripherals (counting consoles, mice and cases) are excluded with the Raspberry $\mathrm{Pi}$. A few embellishments anyway have been incorporated into a few official and informal groups. As indicated by the Raspberry Pi Foundation, more than 5 million Raspberry Pis have been sold before February 2015, making it the top of the line British PC. By November 2016 they had sold 11 million units.

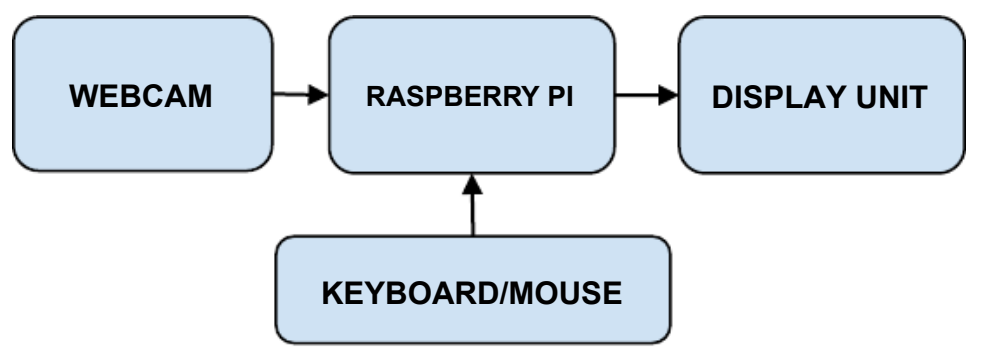

\section{METHODOLOGY}

OpenCV accompanies a coach just as identifier. In the event that you need to prepare your own classifier for any article like vehicle, planes and so on you can utilize OpenCV to make one. Its full subtleties are given here: Cascade Classifier Training.

Here we will manage detection. OpenCV as of now contains numerous pre-prepared classifiers for face, eyes, grin and so forth. Those XML records are put away in opencv/information/haarcascades/organizer. How about we make face and eye locator with OpenCV. 
The framework is modified utilizing Python programming language.We have created three calculations, for face detection from a given picture, from an organizer of pictures and for continuous face detection.

\section{FACE DETECTION FROM A GIVEN IMAGE}

Histogram evening out is done on the information picture. Haar classifier is utilized for picture estimation procedure and once face is distinguished, a red jumping box is drawn on the identified face. Distinguished face and sub appearances are spared and time taken for detection is printed.

\section{Face detection from a folder of images}

After Histogram equalization of the given image, Haar classifier is again used for image calculation process. The difference from the first algorithm is that in addition to saving the detected face to a specified folder, the algorithm also checks if each image belongs to the source directory. If yes, the current file is named as a valid image with the file name. Otherwise, the file is named as an invalid image.

\section{Real Time Face Detection}

Video is caught ongoing utilizing the webcam. Up to a face is distinguished, a red jumping box is drawn and the video is shown in the yield window. The calculation is proficient enough to recognize numerous appearances too.

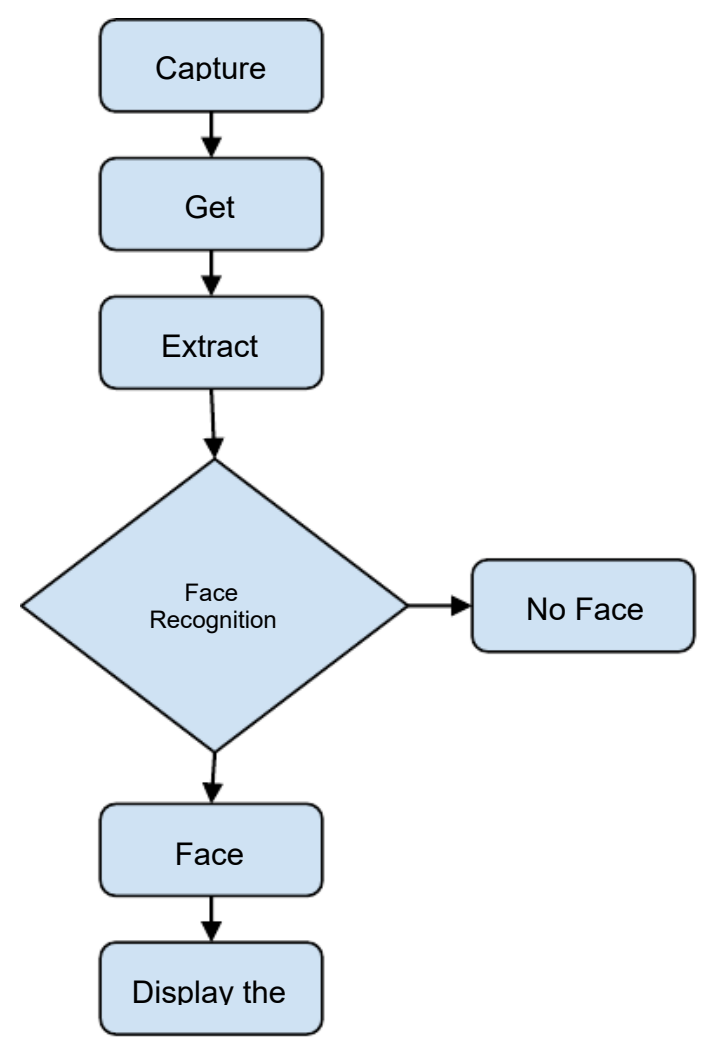

\section{WORKING}

This system is Raspberry Pi 3 module which are competent for preparing of the Live video and picture handling utilizing locally available GPU office. This framework additionally comprise of Webcam used to catch the pictures of client. Utilizing Haar Cascade classifier it is conceivable to distinguish the Face from a Capture Image. First it catching the face and it 
putting into the database. After this it perceive the face dependent on the catch picture and database image.Face detection is worried about discovering whether there are any appearances in a given picture and, if present,returns the picture area and substance of each face. Most face detection calculations are planned in the product space and have a high detection rate, however they regularly require a few seconds to distinguish faces in a solitary picture, a preparing speed that is inadequate for continuous applications. This paper portrays a straightforward and simple equipment usage of face detection framework utilizing Raspberry $\mathrm{Pi}$, which itself is a minicomputer of a charge card estimate and is of an exceptionally low cost. The framework is customized utilizing Python programming language. Both continuous face detection and face detection from explicit pictures, for example Item Recognition, is completed and the proposed framework is tried crosswise over different standard face databases, with and without commotion and obscuring impacts. Proficiency of the framework is examined by computing the Face detection rate for every one of the database. The outcomes uncover that the proposed framework can be utilized for face detection even from low quality pictures and shows amazing execution effectiveness

\section{REFERENCES}

[1] KritJanard and WorawanMarurngsith "Accelerating Real time Face detection on Raspberry Pi telepresence Robot" Fifth International Conference on Innovative Computing Technology (INTECT 2015)

[2] T. Ahonen, A. Hadid, and M. Pietikainen. Face recognition with local binary patterns. In European Conference on Computer Vision,

[3] M. Bicego, A. Lagorio, E. Gross, and M. Tistarelli. On the use of sift features for face authentication. In IEEE International Conference on Computer Vision and Pattern Recognition

[4] M.S. Bartlett, J.R. Movellan, and T.J. Sejnowski. Face recognition by independent component analysis. IEEE Transactions on Neural Networks

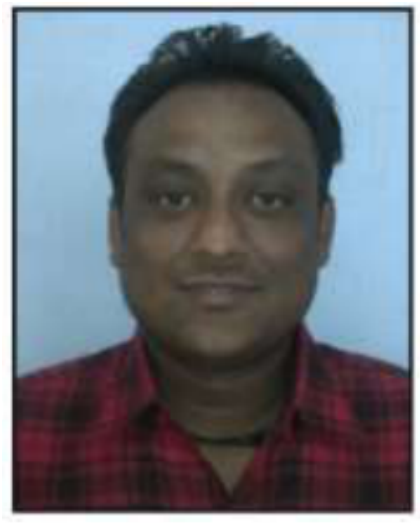

Manav Bansal received his B.Tech. degree in Computer Science from Uttar Pradesh Technical University, M.Tech. degree in Information Technology from Karnataka State Open University, and M.B.A. in Information Technology from Punjab Technical University, M.Phil from Manav Bharti University and pursuing Ph.d. in Computer Science from Monad University. He is working as Assistant professor in Sir Chhotu Ram

Institute of Engineering and Technology, CCS University Meerut. His research interests include Computer Vision, Design and Analysis of Algorithms, Theory of Formal Languages. 